\title{
Guidelines for identification and treatment of individuals with attention deficit/ hyperactivity disorder and associated fetal alcohol spectrum disorders based upon expert consensus
}

\author{
Susan Young ${ }^{1,2^{*}}$, Michael Absoud ${ }^{3}$, Carolyn Blackburn ${ }^{4}$, Polly Branney ${ }^{5}$, Bill Colley ${ }^{6}$, Emad Farrag ${ }^{7}$, Susan Fleisher ${ }^{8}$, \\ Ges Gregory ${ }^{9}$, Gisli H. Gudjonsson ${ }^{10}$, Keira Kim ${ }^{11}$, Kieran D. O'Malley ${ }^{12,13}$, Moira Plant ${ }^{14,15}$, Alina Rodriguez ${ }^{1,19}$, \\ Susan Ozer ${ }^{16}$, Inyang Takon ${ }^{17}$, Emma Woodhouse ${ }^{10}$ and Raja Mukherjee ${ }^{18}$
}

\begin{abstract}
Background: The association of attention deficit/hyperactivity disorder (ADHD) and fetal alcohol spectrum disorders (FASD) results in a complex constellation of symptoms that complicates the successful diagnosis and treatment of the affected individual. Current literature lacks formal guidelines, randomized control trials, and evidence-based treatment plans for individuals with ADHD and associated FASD. Therefore, a meeting of professional experts was organized with the aim of producing a consensus on identification and treatment guidelines that will aid clinicians in caring for this unique patient population.
\end{abstract}

Methods: Experts from multiple disciplines in the fields of ADHD and FASD convened in London, United Kingdom, for a meeting hosted by the United Kingdom ADHD Partnership (UKAP; www.UKADHD.com) in June 2015. The meeting provided the opportunity to address the complexities of ADHD and FASD from different perspectives and included presentations, discussions, and group work. The attendees worked towards producing a consensus for a unified approach to ADHD and associated FASD.

Results: The authors successfully came to consensus and produced recommended guidelines with specific regards to identification and assessment, interventions and treatments, and multiagency liaisons and care management, highlighting that a lifespan approach to treatment needs to be adopted by all involved. Included in the guidelines are: 1) unique 'red flags', which when identified in the ADHD population can lead to an accurate associated FASD diagnosis, 2) a treatment decision tree, and 3) recommendations for multiagency care management.

Conclusions: While clinically useful guidelines were achieved, more research is still needed to contribute to the knowledge base about the diagnosis, treatment, and management of those with ADHD and associated FASD.

Keywords: Attention deficit/hyperactivity disorder (ADHD), Fetal alcohol spectrum disorders (FASD), Co-morbid or associated FASD, Treatment guidelines, Consensus, Interventions

\footnotetext{
* Correspondence: susan.young1@imperial.ac.uk

${ }^{1}$ Imperial College London, London, UK

${ }^{2}$ Broadmoor Hospital, West London Mental Health Trust, Crowthorne,

Berkshire, UK

Full list of author information is available at the end of the article
}

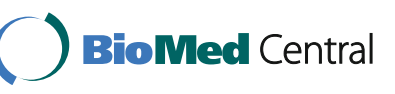

(C) 2016 The Author(s). Open Access This article is distributed under the terms of the Creative Commons Attribution 4.0 International License (http://creativecommons.org/licenses/by/4.0/), which permits unrestricted use, distribution, and reproduction in any medium, provided you give appropriate credit to the original author(s) and the source, provide a link to the Creative Commons license, and indicate if changes were made. The Creative Commons Public Domain Dedication waiver (http://creativecommons.org/publicdomain/zero/1.0/) applies to the data made available in this article, unless otherwise stated. 


\section{Background}

Approximately $5 \%$ of the population world-wide is reported to have a diagnosis of attention deficit/hyperactivity disorder (ADHD) [1], a disorder often associated with co-morbid conditions that can complicate identification and treatment [2]; fetal alcohol spectrum disorders (FASD) are among those conditions that are commonly found to co-exist with ADHD [3]. There is an established knowledge base with regards to identification and treatment guidelines for ADHD, and similarly, yet to a lesser degree, for FASD; there is also an increasing awareness and knowledge base of individuals having both disorders concurrently. There are not, however, formal guidelines for diagnosis, randomized controlled trials of any kind, or evidence-based treatment plans for this unique group of individuals with ADHD and associated FASD [4]. Therefore, this multidisciplinary meeting was organized to produce identification and treatment guidelines for patients with ADHD and associated FASD according to the clinical expertise and knowledge among the attendees based upon consensus.

In this article we refer to the co-existence of ADHD and FASD as-ADHD and associated FASD - and not FASD with associated or co-morbid ADHD because: 1) most alcohol affected individuals present with and are initially evaluated for attention and executive function deficits [3, 5]; 2) management of ADHD symptoms in the FASD individual is integral to treatment and may minimize the potential impact of the common secondary disabilities associated with FASD [3]; and 3) speculation remains as to the nature of the relationship of the co-existing disorders with each other $[3,6]$. The authors recognize that current formal guidelines for the management of ADHD symptoms do not account for the implications of prenatal alcohol exposure in affected individuals, thus the formulation of expanded guidelines for management of ADHD and associated FASD is the focus of this discussion. Firstly, we describe what is known about ADHD and FASD separately and then we describe what is known about the co-existing disorders. Next we discuss how our collaborative efforts led to consensus. Lastly, we present our recommended guidelines for clinicians, with specific regards to: identification and assessment, interventions and treatment, and multiagency liaisons and management. Included are three pertinent case studies that exemplify the need for individualized treatments and services, which commonly extend beyond medical care. A list of abbreviations can be found at the end of the manuscript.

\section{ADHD}

ADHD is a childhood onset, diagnosable neurobiological disorder with genetic and environmental origins [7] characterized by pervasive behavioral symptoms of hyperactivity, inattentiveness, and impulsivity that interfere with functioning and development [8]. In 1968 the
American Psychiatric Association officially recognized hyperkinetic impulse disorder, which has since been renamed ADHD in the second edition of the Diagnostic and Statistical Manual of Mental Disorders (DSM-II). Symptoms present relatively early in childhood and persist over one's lifespan for about half of individuals with childhood ADHD [9, 10]. A systematic review and metaregression analysis reported an overall worldwide-pooled estimate of $5.29 \%$ [11]. Structural brain MRI studies, comparing individuals with ADHD with controls, report overall reductions in brain volume of those having ADHD and reveal the largest differences in cerebellar regions, the splenium of the corpus callosum, total and right cerebral volume, and right caudate; several frontal regions assessed in two studies also showed large significant differences [12]. In addition to academic and social impairments, children with ADHD are at risk for other psychological disorders and about half have additional behavioral disorders [7, 13]. Meta-analyses of 42 international prison studies, based on data derived from symptom-based clinical instruments, reported $30 \%$ of youth offenders and $26 \%$ of adult offenders have clinically diagnosable ADHD [14]. Furthermore, the adult offenders had higher rates of coexisting psychopathology and greater impairment due to mood, anxiety and personality disorders [15].

Clear diagnostic criteria for ADHD are listed in the Diagnostic and Statistical Manual of Mental Disorders, $5^{\text {th }}$ edition, (DSM-5) [8] and the World Health Organization's International Statistical Classification of Diseases, $10^{\text {th }}$ edition, (ICD-10) [16] (note that ADHD is the ICD-10 equivalent of hyperkinetic disorder). While the criteria have not changed from DSM-IV [17], DSM- 5 has updated the definition of ADHD to more accurately characterize the experience of affected adults, and examples have been included to illustrate the types of behavior children, older adolescents, and adults with ADHD might exhibit. There is a strong evidence base in the literature regarding guidelines for identification, treatment, and management of individuals with ADHD, and the authors advocate use of the National Institute for Health Care Excellence (NICE) 2008 guidelines [18].

\section{FASD}

FASD is a non-diagnostic descriptive term referring to the full range of diagnosable conditions caused by the deleterious effects of prenatal alcohol exposure (PAE), including neuropsychological, behavioral, and physical abnormalities [19]. The range of clinical phenotypes varies in severity and outcome depending on the level, pattern, and timing of maternal alcohol consumption $[19,20]$. Fetal alcohol syndrome (FAS) was first described in 1973 [21]. In 1996 the United States' Institute of Medicine delineated useful classification terms including FAS, partial fetal alcohol syndrome (pFAS), alcohol related birth 
defects (ARBD), and alcohol related neuro-developmental disorder (ARND) [22]. It wasn't until 2000, however, that the term FASD was officially introduced [23].

The implications of heavy PAE are long term and pervasive, affecting the individual, mother, family, and community throughout one's lifespan [24-26]. FASD are considered a "hidden disability" because most individuals affected by PAE are not identified until adolescence or adulthood, if at all [27]. Although FAS is estimated to occur in 2 to 7 per 1000 live births in young children in the United States, and up to 68 per 1000 in high-risk populations $[28,29]$, FASD are more prevalent and may occur in as many as $2-5 \%$ of younger school children in the US and some western European countries [30], which means that as many as 6 to 16 million young children in the US may have FASD, based upon the current US population of 320 million [31]. Meta-analyses of children and youth in a child-care system revealed a $6.0 \%$ pooled prevalence of FAS and a $16.9 \%$ pooled prevalence of FASD [32]. A recent study reported a significantly high proportion $(86.5 \%)$ of FASD among foster and adopted youth referred to a mental health center, which were previously mis- or undiagnosed [33]. Furthermore, a retrospective assessment of children in the United Kingdom reported prenatal alcohol exposure rates of $34 \%$ among "looked after children" and $75 \%$ among those awaiting adoption [34]. Brain imaging techniques have shown that PAE causes permanent structural alterations to the brain, and reduced overall volume [35, 36]. Studies have demonstrated damage to the corpus callosum, cerebellar vermis, basal ganglia, as well as perislyvian, orbito-frontal, and parietal brain regions of individuals with FASD [35]. Additionally, a review of individuals with FASD reported a pooled prevalence of $90.9 \%$ having abnormal peripheral nervous system function results [37].

There is a high prevalence of co-morbid conditions in individuals with FASD [37]. Long term studies report that adolescents and young adults with FASD have major problems with adaptive behavior, with high rates of disrupted education (61\%), trouble with the law (60\%), confinement $(50 \%)$, inappropriate sexual behavior on repeated occasions (49\%), and drug and alcohol related problems (35\%) [26]. Deficits in adaptive functioning can often help clinicians identify individuals with prenatal alcohol exposure [26]. Individuals with FASD present with specific behavioral impairments that make them especially vulnerable to manipulation. They are easily coerced into producing false confessions thereby making them more likely to become involved with the criminal justice system [38]; and one study reported $35 \%$ of individuals with FASD have been in jail or prison at some point [39].

The diagnostic criteria for FAS is well established in the ICD-10 and DSM-5, but the diagnostic criteria for
PAE conditions other than FAS are less precise and subject to considerable debate due to lack of available published evidence [40]. Several FASD diagnostic approaches have been designed to promote best practices, but accurate clinical diagnoses remain, for the most part, to be made by expert clinicians who have wider experience with complex neurobehavioral findings. According to the ICD-10 and DSM-5 diagnostic criteria sets, in the absence of confirmed PAE, the signs and symptoms of FAS fall into three categories: 1) a characteristic pattern of facial anomalies (short palpebral fissure, smooth philtrum, thin vermilion border of the upper lip); 2) evidence of growth retardation (pre-and/or postnatal); and 3) evidence of central nervous system (CNS) abnormalities [19-21]. While the presence of facial anomalies enables diagnosis in some children with histories of heavy prenatal alcohol exposure, most PAE affected individuals do not exhibit obvious dysmorphology [41] and/or lack reliable PAE history [42], which greatly hinders identification. Although useful for classification, the IOM's terms are not listed as diagnosable conditions in the ICD or DSM criteria sets. DSM-5 has proposed a diagnostic criteria set for neurobehavioral disorder associated with prenatal alcohol exposure (ND-PAE), but is not yet intended for clinical use. A recent paper on FASD [43] (which thoroughly elucidates the diagnostic terms and includes the proposed DSM-5 criteria on ND-PAE) and recent book on ARND [44], together explain why a more accessible diagnosis is needed. Until the DSM-5 or ICD-11 have established criteria sets, the authors advocate using the 2005 Canadian Guidelines for the Diagnosis of FASD [20]. These guidelines focus more on the neurobehavioral assessment over growth deficiencies and recommend using a multidisciplinary approach to diagnosis [20].

\section{ADHD and associated FASD}

Individuals with histories of heavy PAE or ADHD are at risk for a wide range of impairments including behavioral and neuropsychological deficits [37, 45, 46]. Children with a diagnosis of ADHD present with impairments similar to those apparent in alcohol-exposed children especially with regards to executive functioning and attention deficits [47]. There are, however, clear distinctions between the two groups; individuals with ADHD with PAE perform worse on conventional tests sensitive to attentional problems and conduct disorder, compared with individuals with FASD [46]. ADHD is the most common psychiatric disorder diagnosed in children with PAE in the United States at a rate of $41 \%$ [48] and in individuals with FASD world-wide at a rate of $48 \%$ [49]. Given the range of a 2$5 \%$ prevalence rate of FASD in the US and some western European countries and the $41-48 \%$ prevalence rate of co-occurring ADHD, we estimate the prevalence of ADHD and associated FASD to be $0.8-2.4 \%$ among 
children in the US and some western European countries. Although, because many affected individuals are un-diagnosed, we speculate that the two disorders occur together at far higher rates than estimates suggest. Despite the depth of knowledge on the separate disorders and an increasing amount on the concurrence, the exact relationship between the two remains unclear and there is a debate over whether or not the presentation necessarily constitutes separate entities [3, 6, 25]. Furthermore, evidence indicates that ADHD symptoms in individuals identified with FASD may be a specific clinical phenotype [3]. Understanding the relationship between the disorders is complicated by the fact that ADHD is primarily defined descriptively (without a clear etiology) and FASD are primarily defined mechanistically (with a clear etiology). The clinical quality of ADHD in children with FASD often differs from that of children having only ADHD [50]; behavioral studies report exacerbated effects of having both PAE and ADHD compared with alcohol exposure alone [45]. The majority of individuals with PAE lack the physical and facial dysmorphology commonly associated with FAS [41] and many lack reliable histories of PAE [42], which further increases the difficulty in making a clear diagnosis. According to the National Organization of Fetal Alcohol Spectrum (NOFAS), most individuals affected by FASD are often only identified - if at all- subsequent to a referral for learning disabilities or for cooccurring ADHD [27], likely resulting in many mis- or undiagnosed individuals. In the absence of high quality studies examining the relationship between ADHD and FASD and reporting on effective treatments, the authors have produced identification and treatment guidelines for clinicians, based upon expert consensus.

\section{Methods}

Experts from multiple disciplines in the fields of ADHD and FASD convened on the $27^{\text {th }}$ of June 2015 in London, United Kingdom, for a meeting hosted by United Kingdom ADHD Partnership (UKAP; www.UKADHD.com). The meeting included presentations with electronic slides, discussions, and group work; and was recorded and later transcribed. Psychiatrists, neuro-developmental pediatricians, psychologists, education experts, researchers, and others having personal experience with the impact of ADHD and/ or FASD, engaged in lively thought provoking discussions throughout the day, with the aim of reaching consensus.

The meeting commenced with five presentations (including case studies) on the following topics, where each was followed by a question and answer session:

- ADHD and FAS: The Clinical Presentation

- Alcohol Exposure and Risk for Development of FAS

- Psycho-pharmacotherapy Interventions for Co-morbid ADHD/FAS
- Education Interventions for Co-morbid ADHD/FAS

- The Criminal Justice Perspective

Following the presentations, all attendees separated into three breakout session groups (smaller subsets of the meeting attendees) to produce a framework of guidelines with specific regards to:

- Identification and Assessment

- Interventions and Treatments

- Multiagency Liaisons and Care Management

The discussions during the breakout sessions were facilitated by group leaders and summarized by note takers. The group leaders then presented their group's framework of guidelines in a feedback session to all the meeting attendees for another round of discussion and debate. The medical writer consolidated the meeting transcription, electronic slide presentations, and breakout session notes into the manuscript. Lastly, the meeting transcription and manuscript were circulated to all authors for review to ensure agreement and a final consensus for a unified approach to ADHD and associated FASD was reached.

\section{Results}

\section{Discussion}

The authors successfully produced identification and treatment guidelines for children and adults with ADHD and associated FASD, to aid clinicians in caring for this unique patient population, and the results follow below. Due to the nature of the co-existing disorders, it was recognized that services beyond medical care are required for the vast majority of patients.

\section{Identification and assessment guidelines}

When considering the possibility of ADHD co-existing with FASD it is essential to investigate, with hopes of obtaining, an accurate and detailed PAE history. It is also important to routinely screen for FASD when ADHD is identified or for ADHD when FASD is identified; the DSM-5 and ICD-10 criteria sets are to be utilized for diagnosing ADHD and FAS, and we advocate the use of the 2005 Canadian guidelines for diagnosing all other FASD until the DSM-5 and ICD-11 criteria sets are further established. It is important to recognize the unique characteristics or 'red flags' that may be present in the individual with ADHD, which may assist in the identification of co-existing FASD. Prevalence rates of ADHD are known to be high in certain high-risk populations such as prisoners, 'looked after' children, adoptees, and individuals born prematurely. Efforts should be made among medical, social, educational, and criminal justice services to increase awareness of the need for appropriate referrals for individuals in these high-risk groups. They 
should be referred to suitable local pathways for either ADHD or FASD, based upon their primary presenting condition. It is recognized that individuals with subtle presentation may fail to meet the threshold for ADHD and are likely to be missed with this approach; ideally, the development of locally based complex neuro-developmental services is recommended. Neuro-developmental services could help identify and further assess affected individuals, which could potentially minimize the secondary health and social disabilities associated with ADHD and FASD.

\section{The clinician should carefully identify and assess PAE history}

Maternal alcohol use during pregnancy should be included in a standard interview for those being assessed for ADHD. The World Health Organization's Alcohol Use Disorders Identification Test offers a simple method of screening for excessive drinking and assists in making a brief assessment [51]. It is recognized that clinicians, patients and their families may be uncomfortable with this line of questioning; therefore it is recommended that enquiries concerning possible PAE be incorporated in the context of obtaining a detailed family history, in order to 'normalize' the questioning for both the clinician and the individual under assessment. Additionally, it is recognized that mothers may find difficulty in admitting retrospectively that their child suffered PAE, possibly as a result of perceived stigma. It is also important to ascertain the accurate amount of alcohol consumed during pregnancy-depending on the size and alcohol content of the drink, two drinks per day could equate to two or more units. Obtaining an accurate alcohol use history will likely depend upon the interviewer's skill and training. As a guide, the clinician should investigate typical pre-pregnancy lifestyle factors including general drinking behaviors, use of prescription medication, and other drugs. As part of this investigation the clinician may need to access historical records from social workers, midwives, and/or obstetricians. The lack of honest and accurate estimates of alcohol consumption combined with the oft-times lack of availability of the mother [52] is problematic and can result in large groups of potentially high-risk individuals remaining unidentified. Best practice therefore suggests that countries adopt standardized investigative procedures to identify and track these potentially at risk individuals and their families. Due to the sensitive nature of obtaining an accurate history of drinking during pregnancy, an empathic, non-judgmental approach to questioning is essential.

\section{ADHD or FASD history and additional screenings}

If the child or adult has been previously diagnosed with ADHD then the clinician should routinely screen for FASD and likewise FASD for ADHD. A diagnosis of FAS should be made according to DSM- 5 and ICD-10 criteria sets and all other FASD should be assessed using the 2005 Canadian guidelines. A diagnosis of ADHD should be made according to DSM-5 and ICD-10 criteria sets, utilizing standard screening and diagnostic tools: Conners' Rating Scales; Swanson, Nolan, and Pelham-IV (SNAPIV); and ADHD Rating Scale-IV. It is recommended that the affected individual be referred, when appropriate, to neuro-developmental services for additional assessments in adaptive behavioral functioning, executive function, and communication abilities.

\section{Unique characteristics of ADHD with associated FASD}

We recognize a number of unique characteristics or 'red flags', which when present in individuals with ADHD, may assist in the identification of associated FASD. These 'red flags' can be considered as potential risk factors for having FASD. Clinicians are to be alerted if any of the following unique characteristics are identified in patients with ADHD:

- Possible or confirmed history of maternal alcohol consumption during pregnancy,

- Predominant presentation with inattentive subtype of ADHD, in addition to some impulsive behaviors,

- Failure to respond to, or increased behavioral disturbance when prescribed methylphenidate,

- Poor psychostimulant response in children with IQ under 50,

- Physical indicators of FAS or pFAS, including delayed growth and/or specific facial features (e.g., smooth philtrum, thin upper lip, and small eye openings), and/or a

- Lack of response to typical behavioral interventions.

\section{Intervention and treatment guidelines}

Treatments for ADHD and associated FASD require an individualized approach, as do all neuro-psychiatric disorders. Clinicians should refer for multi-modal assessments (often involving parents, teachers, occupational therapists, and speech and language therapists), when necessary, to assist them in treatment plan development. The Centers for Disease Control offers guidelines to service providers in making referral decisions specific to individuals with FAS [41], but can be a good resource for referral considerations for individuals with ADHD with associated FASD. When developing the treatment plan the primary rearing environment should be recognized and evaluated because it has different modulating effects on affected individuals. The following co-occurring problems must be considered as contributors to the clinical presentation; early onset post-traumatic stress disorder due to direct or indirect exposure to violence including specific alcohol related violence, and/or reactive or 
disorganized attachment disorders due to problems with adoptions or multiple care givers (as in sharing parenting arrangements between foster and birth families). Furthermore, because a combination of non-pharmacological and pharmacological treatments are most often required when treating $\mathrm{ADHD}$, it is recommended that this also be the case for individuals with associated FASD-noting that pharmacological treatments should ideally be administered secondary to nonpharmacological (psychological and behavioral) therapies. While treatments differ between ADHD with and without associated FASD, the 2008 NICE treatment guidelines for ADHD are mostly applicable to this group and should be used as a basis on which to build the treatment plan and the medication options available for ADHD are to be considered in the context of PAE. If other co-morbidites are unveiled throughout the assessment process, then it will be necessary to modify the treatment plan. NICE guidelines allow for treatment with medication after 5 years of age following psychological and behavioral therapies and state that medication can be considered as the first option for individuals with a severe presentation of ADHD. Individuals with ADHD and associated FASD (where hyperactivity, rather than a sensory seeking need, is a predominant feature) will likely be categorized as having a severe presentation of ADHD and therefore may be treated with medication as a first option upon diagnosis. Because ADHD and associated FASD affects individuals at a very young age with symptoms persisting over their lifespan, it is crucial that they are treated, monitored, and supported throughout their entire life. Additionally, it is important to maintain a non-judgmental attitude towards affected individuals and their families while developing and delivering the suitable treatment plan. We developed a treatment decision tree (Fig. 1) unique to children, adolescents, and adults with ADHD and associated FASD that outlines and supplements our guidelines.

\section{The clinician should administer the following treatments}

Non-pharmacological treatments for the family

Family support is usually necessary, and oftentimes special support may be required for the birth mother, because she may need therapy to cope with possible feelings of guilt and shame relating to alcohol use during pregnancy. Educating and encouraging the birth mother may result in reduced or no alcohol use in subsequent pregnancies. It is also important to recognize the particular needs of adoptive parents and additionally to recognize the ongoing impact of the child's condition on the family because they may need support groups or respite services.

\section{Non-pharmacological treatments for the child}

Treatment should be delivered primarily via the parents and/or care givers by educating them in healthcare and child training strategies, and techniques about the best ways to care for and help their child. It is important for affected children, and especially for those with additional co-existing learning difficulties, to be fully assessed. It may be necessary to develop a formal individualized educational plan to access additional services to address the need for educational supports.

\section{Non-pharmacological treatments for the adolescent and adult}

Treatment can be delivered directly to the individual. With regards to treatment decisions, however, it must be first established whether or not the individual has the capacity to make such decisions and whether or not it is appropriate to have the family involved as part of a shared plan or agreement. Safeguarding issues need to be taken into account when dealing with children, adolescents, and vulnerable adults. These individuals are capable of attending and benefiting from group treatment/therapy sessions. Symptoms and their associated impairments can become more marked in older children as they progress into adulthood, thus key topics that should be included in therapy, group or otherwise, are: risk taking behaviors, inappropriate sexual behaviors, problem solving skills, social skills, and sleep hygiene techniques. It is of high importance to note that risk taking behaviors and problem-solving deficits in combination with impulsivity may increase the risk of suicide.

\section{Pharmacological treatments when psychological and behavioral interventions are inadequate}

It is important to carefully monitor progress and measure the effects of non-pharmacological treatments through goal-based outcomes. If the co-existing disorders are still causing significant impairment, after a short period of one to three months, then a progression to the use of medication should be made. It is recognized that family involvement with regular and frequent follow-up visits are essential to successful pharmacological treatment. If a child resides within a chaotic family it will be especially necessary to assess whether a responsible adult is correctly administering the medication.

\section{Pharmacological treatments following baseline physiological tests}

The need to physiologically assess the individual and run baseline tests is emphasized as highly important for this group. The NICE treatment guidelines for ADHD recommend the monitoring of blood pressure, 


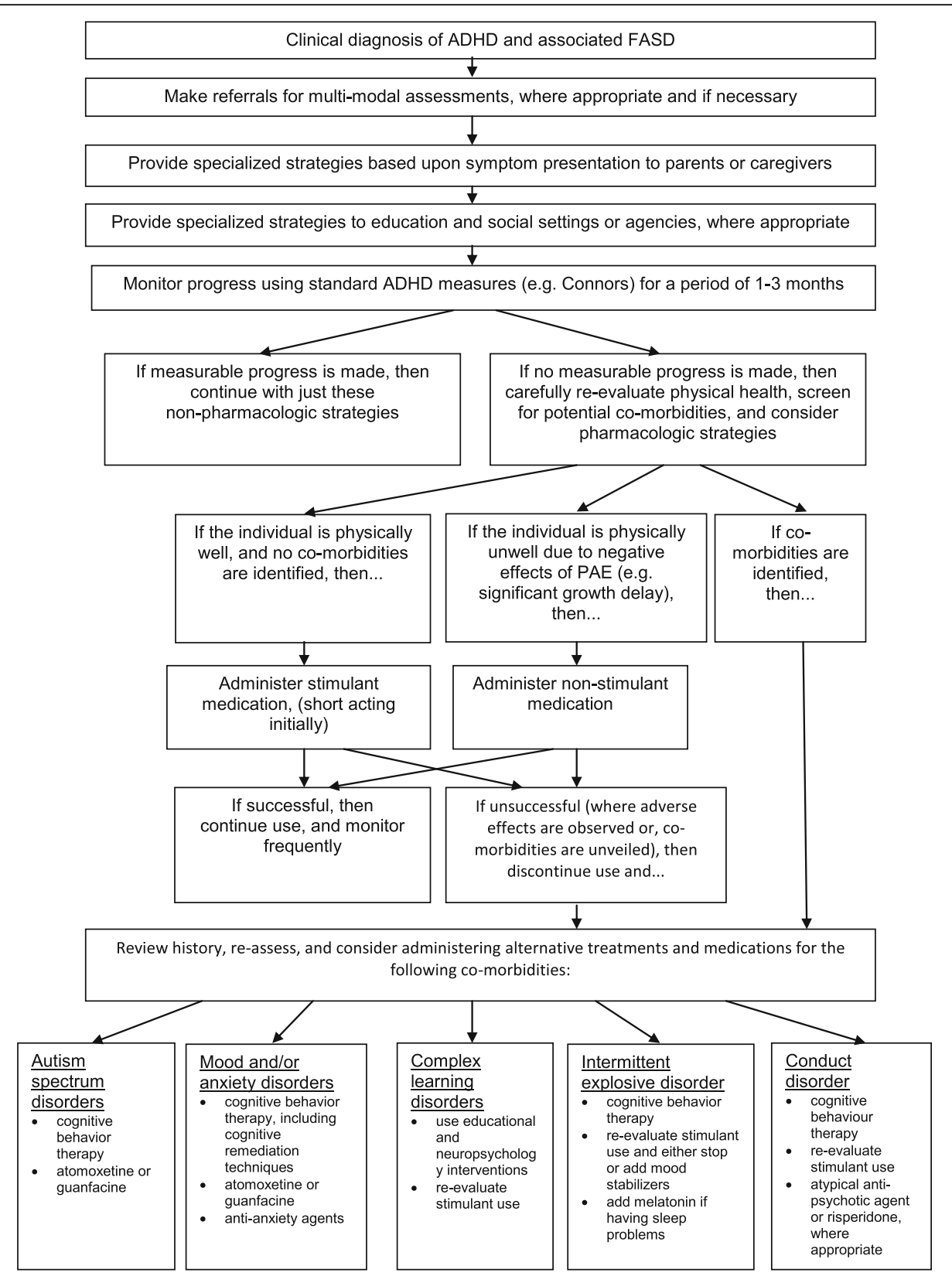

Fig. 1 Treatment Decision Tree

pulse, height, weight, and the investigation of cardiovascular history with an ECG prior to medication use. The following additional baseline tests should ideally be ordered, to investigate the possible damaging effects of PAE: full blood count (FBC), thyroid function test (TFT), liver function test (LFT), blood sugar, folate, gamma-glutamyltransferase (GGT; a test specific to alcohol, especially important for teenagers), blood pressure, and renal function test (RFT); these baseline tests should be repeated if indicated clinically. Additionally, care should be taken to obtain a full clinical seizure history, if indicated clinically.
Pharmacological treatments to improve attention and reduce hyperactivity and impulsivity

Currently developed medication can treat the symptoms of ADHD, but cannot cure the disorder. Medication can improve attention and reduce hyperactivity and impulsivity, thereby allowing the individual to effectively manage their difficulties, access and engage with suitable therapies, and build upon their skills. Management of the core symptoms may improve learning, minimize their potential impact, and ameliorate the common secondary disabilities associated with FASD. Case study 1 (see Fig. 2) reveals the complex nature of the assessment process and demonstrates 
how a carefully managed treatment plan can have a positive impact on affected individuals.

\section{Pharmacological treatments with careful regards to dosage and side effects}

The side effects of medication use, among individuals with ADHD and associated FASD, may be similar when compared with individuals with ADHD, but more marked due to the abnormal physiological aspects of the disorder. Some stimulant medications can lead to more pronounced anxiety, appetite, and sleep problems; they may also affect growth hormones and may increase the prevalence of tics. The response to medication can be idiosyncratic and unpredictable because some medications may cause a regression of behavior. In particular, high doses of atomoxetine (ATX) may cause liver damage, therefore low doses are recommended initially. Before increasing a medication dosage it is recommended that the non-pharmacological strategies are revisited and additional assessments are made to account for any under or misdiagnosed coexisting conditions. The individual should be re-evaluated and monitored frequently throughout the intervention process.

\section{Pharmacological treatments with careful regards to the risks of developing an addictive disorder}

Short-acting stimulants are typically trialed first in patients with ADHD to examine tolerance before progressing to

'Alison' is a 9 year old happily living with her adoptive parents. She was adopted at 22 months of age. Hospital notes confirmed maternal alcohol use in pregnancy, and a pediatric neurologist diagnosed her with residual encephalopathy with syndrome of liquor hypertension, increased neuroexcitabilty, and psychosocial speech development impairment. She did not present clinical problems on post adoption examination, but at 7 to 8 years of age had academic and behavioral school problems because of pervasive distractibility, lack of attention, and impulsivity (including inappropriately blurting things out).

An intellectual assessment showed verbal and performance IQ in the average range but a large scatter in the test scores. The psychologist reported a 15 point differential between verbal comprehension and working memory. The Vineland Adaptive Behavioral Scales showed Daily Living Skills at the $10^{\text {th }}$ percentile, Socialization at the $10^{\text {th }}$ percentile, and Communication at the $30^{\text {th }}$ percentile.

Alison did not reveal any facial dysmorphic features, height and head circumference were in the $50^{\text {th }}$ percentile, but weight was in the $2^{\text {nd }}$ to $10^{\text {th }}$ percentile. The adoptive parents reported that her weight had always been low. Gross and fine motor assessment showed evidence of balance problems with both truncal and gait ataxia. Sensory examination was normal.

On further clinical examination, Alison demonstrated a good basic understanding of the general knowledge on the examination tests, however, she had major problems with auditory retention and comprehension and additional problems with visual retention and comprehension; there was also evidence of dyscalculia. Alison had no significant expressive language problems, although in the assessment sessions she did have a tendency to become disconnected at times, due to pervasive distractibility and lack of concentration. She was observed to be quite disorganized in play and impulsive at times, however, she did not demonstrate physical hyperactivity.

Alison was diagnosed with neurodevelopmental disorder prenatal alcohol exposure, Code 315.8 (DSM 5), with clinical presentation of ADHD, primarily inattention, code 314.00 , and evidence of developmental coordination disorder, code 315.4. Both parent and school Conners' Rating Scales were completed following the first assessment visit. Following diagnosis, a document providing specialized management strategies was given to her parents.

After carefully evaluating her baseline blood tests and echocardiogram, three weeks later Alison was treated with atomoxetine for her ADHD symptoms. Atomoxetine was chosen due to Alison's low weight and the relative contraindication of dextroamphetamine. In only the first 4 weeks of therapy, Alison's parents reported a good response, with improved behavior. She will continue to be monitored for correct dosaging and for improvement in school.

Alison's actual name, age, and gender have been changed to protect her anonymity.

Fig. 2 Case Study 1—Pharmacological Treatment 
long-acting stimulants. This treatment can be problematic, however, in adolescents with ADHD and associated FASD because short-acting stimulants may potentially contribute to the development of addictive disorders during the teenage years. Therefore, long-acting/biphasic stimulants or atomoxetine (which do not give an immediate 'buzz') are recommended because they are less likely to contribute to the development of an addictive disorder.

\section{Multi-agency liasons and care management}

The effects of ADHD and associated FASD are lifelong and pervasive, affecting the individual, mother, family, and community. Therefore, it is extremely important that interventions are comprehensive, continue throughout the lifespan, and extend beyond medical care to include when necessary: social, educational, mental health, employment, and criminal justice services. These interventions are likely to be productive when service agencies collaborate with each other. The coordinated efforts of service professionals should support not only affected individuals, but their families as well. In addition to multiagency collaboration, it is important that each agency offers distinct services designed to enhance quality of life and long term outcomes. While the non-pharmacological treatments previously mentioned cover some of these aspects, there are many additional services that may need to be accessed and managed at different phases of life.

\section{The clinician should make referrals for the following services}

\section{Social services for the family}

If there is suspected or confirmed PAE and/or subsequent failure to engage with professionals, social service referrals for the mother and developing child may need to start prior to birth. Because affected children often display challenging behaviors, social service professionals can offer parents, caregivers, and siblings, training and/ or support for managing those behaviors, thereby bolstering family resilience. Family resilience is also likely to be enhanced with the provision of respite services and group support. Social service professionals should routinely review the needs of the family, including possible financial needs, in order to provide timely and comprehensive services, which is especially important during key transition times.

\section{Social services for the young child}

Young children would benefit from preschool placements, where staff have an understanding of FASD and ADHD, and can offer an appropriate environment to meet the developmental needs of the child; and may be referred for specialized stimulating play therapy and/or other preschool services. It may be required that pediatric medical, mental health, social care, and educational professionals coordinate their services (with parental input) to ensure coherent and consistent support. It is important to recognize the potential need for intervention by local authorities or the need for a referral to an adoption specialist. Given the high incidence of placement breakdown, and the subsequent impact of repeated failure on the child and care system, commissioning officers should carefully evaluate the relative merits of foster and adoption placements against more specialized and perhaps resilient services. When children with ADHD and associated FASD are separated from their birth mothers and moved through the care system, they are often inaccurately identified as having insecure or disorganized attachment disorders, instead of being accurately identified as having developmental, emotional, and behavioral difficulties attributed to PAE. In addition, safeguarding issues should be assessed and, in cases of abuse and exploitation, child protection services should be informed and accessed.

\section{Social services for the adolescent}

Social services may need to be accessed because puberty is a particularly vulnerable phase of development. Many young people affected by PAE may crave friendship and a sense of belonging, but often lack the skills required to achieve these. Supported social groups may therefore assist in establishing meaningful relationships with peers within the local community, which is thought to be a protective factor in averting deterioration in mental health, and enhance quality of life. It is important to recognize breakdowns and/or transitions in living accommodations, education placement and treatment plans for these young adults. It is also important to recognize the increased risk of drug and alcohol abuse or misuse during adolescence. The clinician may have a key role regarding safeguarding issues by screening for abuse and exploitation, and accessing child protection services if necessary.

\section{Educational services for the preschool child}

Additional support for the child may be necessary starting in preschool. It is important to recognize the need for child friendly, age-appropriate tasks and activities. It is also important that young children receive broad assessments of needs across multiple measures, and particularly in language comprehension. The pre-school and young primary school child, and family, may benefit from home-visits from educational services to help parents to understand the developmental benefits of learning through play, methods for encouraging and enhancing verbal and non-verbal communication, and in managing challenging behaviors.

\section{Educational services for the primary school child}

It is important to obtain a comprehensive assessment of needs and functional analysis of behavior, and to 
consider the possible impact of deprivation and limitations unique to these children. It may be necessary to make a referral for the development of an individualized education plan with track-able goals (key learning and developmental targets attainable within a specified timeframe) to document progress. Education planning should include input from students themselves, parents and/or caregivers, and teaching professionals. The assessments and individualized education plan are helpful in determining the student's best learning environment (e.g., inclusive mainstreaming or special education) and may be required to access education supports and appropriate placement. It is essential for all involved to recognize that these students often benefit most from a strengths-based approach and from differentiated learning. Teachers and educational psychologists will most likely require training to develop relevant assessments and educational strategies specific to this population. Staff awareness, understanding, and competence are key factors in a student's progress.

Teaching professionals need to consider not only the curricular and staffing requirements of affected students, but also their sensory and social needs. Teachers and support staff need to identify the student's possible barriers to effective engagement with peers (e.g., social communication and an understanding of 'friendship'), and provide supportive environments in which social skills may be developed or enhanced. Individuals having difficulty with arousal and mood self-regulation may find mainstream settings too overwhelming and may benefit from a more supported special education setting. Nevertheless, even severely affected individuals can be successfully mainstreamed with support (see case study 2; Fig. 3). Challenging behaviors and/or a disengagement from learning often arise when students are not placed in the appropriate setting. Teachers and support staff should assess challenging behavior from a functional, rather than judgmental, perspective and be sensitive to the potential impact that their own responses may have on a student when difficulties arise. Punitive measures are less likely to be effective than strategies based on positive behavior management.

\section{Educational services for the adolescent and adult}

Educational supports are likely needed when there are breakdowns and/or transitions in placement in secondary school or higher education and when the individual shows behavioral signs of being disengaged from schooling. Because school is a moderating influence, the premature cessation of formal learning is likely to elevate the risk of offending behavior, which can result in a decline in long term mental health. Furthermore, family resilience is also negatively impacted by the school exclusion or placement breakdown. Educational planners must therefore be responsive to the dynamic nature of student's individual needs and make appropriate provisions.

\section{Employment services}

Affected teenagers and adults will likely require help in finding and maintaining employment, but with proper support can engage in meaningful employment. Employment services should be aimed at enhancing skill development, thereby increasing job prospects and sustainable engagement in work. Post-school transition plans should consider the educational and developmental level (rather than chronological age) of the young person. Affected individuals may require staged transitions into employment via work experience, supported with mentors or job coaches, and college link courses in order to fulfill their vocational potential. The identification of specific barriers (e.g., in establishing effective work-place relationships) may be more beneficial than the provision of generic support.

\section{Criminal justice services}

The clinician may have a role in helping affected children or adults by recommending an appropriate representative to interface with the criminal justice system and/or by referring for the development of an identification card indicating the presence of a disability that the affected individual can present to criminal justice service personnel if necessary. According to the National Organization on Fetal Alcohol Syndrome, "The criminal justice system can help FASD-affected individuals by: educating judges, lawyers, and parole officers about the characteristics and behaviors of individuals with FASD, establishing a screening and referral process for those with ADHD and FASD who enter the juvenile justice or adult criminal justice system, establishing/utilizing alternative sentencing programs for affected individuals who have committed nonviolent offenses, offering referral information for the children of incarcerated women who may have been prenatally exposed to alcohol." [27]. These affected individuals should be diverted, whenever appropriate, into interventional services with the goal of support and skill development to improve functioning in society.

Case study 3 (see Fig. 4) illustrates how a failure to recognize and diagnose FASD could result in a miscarriage of justice. People with FASD are likely to require the services of a 'registered intermediary' in court to facilitate effective communication, and in some cases also during police questioning. As a minimum, like young persons with ADHD, they should have an 'appropriate adult' present during police interviews in order to ensure fairness and justice [53].

\section{Consensus and Conclusions}

Although more research is needed to identify, treat, and manage affected individuals, the authors successfully 
Jade is a young woman, age 19 years old, with multiple co-existing diagnoses including FAS, ADHD, Autistic Spectrum Disorder and Oppositional Defiance Disorder. Jade can be described as: aloof, over excited, easily distracted, anxious, fearful, obsessional, impulsive, noisy, boisterous, overactive, and with high levels of disability in all settings. Her levels of functioning are poor across a range of skills as shown in the figure below. Given her lack of social understanding she is vulnerable to abuse and, possibly, to abuse others.

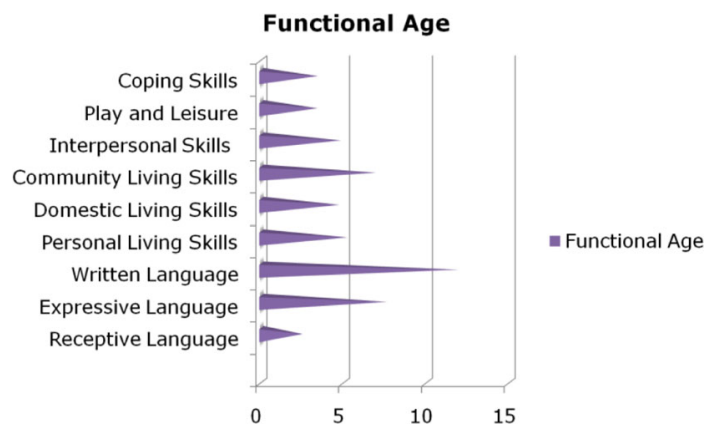

When Jade was 13 years old she attended an Autistic Spectrum Condition Unit attached to a mainstream secondary school. Most of her lessons occurred in mainstream provision, with individual programs delivered in the Unit as necessary. She also had access to music and occupational therapy through her placement in the Unit combining a therapeutic approach with a differentiated national curriculum.

Jade was supported in all lessons by at least one teaching assistant, although for some practical lessons such as science, it was necessary to provide 2:1 adult support. Jade easily became overwhelmed by equipment (such as Bunsen burners), bright liquids and noisy experiments. When over stimulated, she would engage in risky behaviors by touching potentially dangerous equipment (Bunsen burners, ovens, chemicals) and/or quickly move around the room before a member of staff could intervene; and in noisy locker rooms over stimulation led to her climbing on top of equipment such as lockers. While these episodes were sometimes mis-interpreted by some staff as behavioral issues, resulting in sanctions, the support of the specialized teaching staff helped her successfully access and navigate through a mainstream setting. Jade, although previously excluded from a local 'further education' college, is currently planning to study Media and TV at a different college.

Jade's story is further described in the following article: http://www.specialworld.net/2015/09/01/just-one-wont-hurt/. Jade gave consent to sharing her story for this article and Special World Magazine gave permission for the above figure to be reproduced in an open access journal.

Fig. 3 Case Study 2-Secondary Education Provision

came to a consensus for a more unified approach to ADHD and associated FASD. The authors agreed that there is a need to increase the number and training of clinicians and to expand services for this unique population. The authors also agreed upon the need to develop complex neuro-developmental services that could help identify and further assess affected individuals, and intervention services that could help divert affected individuals away from the criminal justice system, when appropriate. It is likely that governmental support will be necessary in the development of these services.
The most significant outcome of the collaborative efforts amongst the multi-disciplinary professionals was the production of clinically meaningful guidelines, based upon consensus. Included in the guidelines are unique 'red flags', which when identified in the ADHD population can lead to an accurate associated FASD diagnosis, a treatment decision tree, and recommendations for multi-agency care management. Given the lifelong, pervasive nature of the associated disorders, the authors' emphasized that a lifespan approach to treatment needs to be adopted by all involved. The implementation of these guidelines 
Susan Burdett, a 39 year old woman, was raped and murdered at her home in New Zealand in March 1992. A year later Teina Pora, aged 17, was in police custody for theft and voluntarily implicated two innocent men in an attempt to receive designated reward money. During police questioning and a visit to the crime scene, Mr. Pora gradually implicated himself in the case. Crime scene DNA did not match Mr. Pora's and the police knew that there had been another man there, but Mr. Pora was never able to name the real perpetrator even though the police had requested this several times.

Mr. Pora was convicted for the murder of Ms. Burdett in 1994, and in 1998 the crime scene DNA was matched with that of a serial rapist, Mr. Malcolm Rewa, who was subsequently convicted. Mr. Pora had a re-trial in 2000 but was convicted again. In 2012, at the request of the defense, Professor Gisli Gudjonsson conducted a detailed evaluation of the case in order for his case to be reviewed. Mr. Pora proved to be abnormally suggestible throughout the evaluation process.

In March 2015 the Privy Council in London quashed Mr. Pora's conviction on the basis of written evidence from two experts, Dr.'s Valerie McGinn and Andrew Immelman, who stated that Mr. Pora suffered from a previously undiagnosed Fetal Alcohol Spectrum Disorder (FASD)-more specifically Alcohol Related Neurodevelopmental Disorder (ARND). Mr. Pora has no relevant facial features but a mild growth deficit. Dr. McGinn's neuropsychological evaluation showed that Mr. Pora had significant deficits in the domains of cognition, adaptive function, memory, executive function and scholastic function. Mr. Pora also showed significant impairment with verbal comprehension and verbal memory, whereas his non-verbal IQ and visual memory were intact.

The available evidence suggests that people with FASD are more suggestible than people with ADHD. They are also more easily manipulated and exploited.

Mr. Pora's story is discussed in detail in the following book: Bennett, M., In Dark Places. The Confessions of Teina Pora and an Ex-cop's Fight for Justice. 2016, Auckland, New Zealand: Paul Little Books.

Fig. 4 Case Study 3-Criminal Justice System

has the potential of making a significant, positive impact on affected individuals.

\section{Abbreviations}

ADHD: Attention deficit hyperactivity disorder; ARBD: Alcohol related birth defects; ARND: Alcohol related neuro-developmental disorder; CNS: Central nervous system; DSM: Diagnostic and statistical manual of mental disorders; FAS: Fetal alcohol syndrome; FASD: Fetal alcohol spectrum disorders; ICD: International classification of diseases; IOM: United States' institute of medicine; ND-PAE: Neuro-developmental disorder associated with prenatal alcohol exposure; NICE: United Kingdom's national institute for health care excellence; NOFAS: United States' national organization of fetal alcohol syndrome; PAE: Prenatal alcohol exposure; pFAS: Partial fetal alcohol syndrome

\section{Acknowledgements}

We acknowledge the assistance of Ben Greer, Hannah Mullens, and Rafael Gonzalez who attended the consensus meeting and made notes during the breakout sessions.

\section{Availability of data and materials}

Not applicable.

\section{Authors' contributions}

SY and RM contributed to the planning and scientific input of this consensus statement. All authors (except KK) attended the consensus meeting. KK interpreted the meeting results and wrote the first draft with input from SY, RM, BC and GHG. KK completed subsequent drafts of the manuscript with input from all authors. All authors have read and approved the final manuscript.

\section{Competing interests}

SY has received honoraria for consultancy, sponsorship for attendance at scientific meetings, educational talks and/or research awards from Janssen, Lilly, Novartis, HB Pharma, Flynn Pharma and/or Shire. GHG has received consultancy fees, speaker fees and/or travel honoraria from Lilly, Janssen and Shire. RM has received speaker fees from Shire, Flynn Pharma, and Lilly. MA has received honoraria for consultancy and/or research awards from Neurim Pharmaceuticals, Novartis, MS Society, Action Medical Research Charities, Shirley Foundation and NIHR. CB has received honoraria for consultancy, sponsorship for attendance at scientific meetings, educational talks and/or research awards from the European Association for Persons with Disabilities, Fosterline England, Winston Churchill Memorial Trust, Positive Path International and Babcock International, The Department of Education, Worcestershire Local Authority, Specialist Schools and Academies Trust and a number of English primary and secondary schools. KO'M has received royalties for authorship of two books on FASD. SO has received honoraria for consultancy and/or educational talks from Flynn Pharma and Shire. MP has received honoraria for educational talks and/or research awards from the World Health Organization, the European Union, the Welcome Trust, the beverage alcohol industry and Shire. IT has received honoraria for consultancy, sponsorship for attendance at scientific meetings and/or educational talks from Shire and Flynn Pharma. The remaining authors have no disclosures.

The meeting was funded by the UK ADHD Partnership (UKAP). Aside from $\mathrm{KK}$, none of the authors received funds for their involvement in developing this consensus.

Consent for publication

The story described in Case study 2 is published with the consent of the patient. 


\section{Ethics approval and consent to participate}

Not applicable.

\begin{abstract}
Author details
${ }^{1}$ Imperial College London, London, UK. ${ }^{2}$ Broadmoor Hospital, West London Mental Health Trust, Crowthorne, Berkshire, UK. ${ }^{3}$ Children's Neurosciences, Evelina London Children's Hospital at Guy's \& St Thomas' NHS Foundation Trust, Kings Health Partners Academic Health Science Centre, London, UK. ${ }^{4}$ Centre for the Study of Practice and Culture in Education, Faculty of Health, Education and Life Sciences, Birmingham City University, Birmingham, UK. ${ }^{5}$ Oxford ADHD Centre, Headington, Oxford, UK. ${ }^{6} \mathrm{CLC}$ Consultancy, Dunkeld, Scotland. ${ }^{7}$ Sussex Partnership NHS Foundation Trust, Children \& Young People's Service, Tunbridge Wells, Kent, UK. ${ }^{8}$ National Organisation for Foetal Alcohol Syndrome-UK (NOFAS-UK), London, UK. ${ }^{9}$ Integrated Child Health, Cambridge and Peterborough Foundation Trust, 80 Thorpe Road, Peterborough PE3 6AP, UK. ${ }^{10}$ King's College London, Institute of Psychiatry Psychology and Neuroscience, London, UK. ${ }^{11}$ Contracted Medical Writer, San Diego, CA, USA. ${ }^{12}$ Child and Adolescent Psychiatrist, FASD Specialist, Slievemore Clinic, Dublin, Ireland. ${ }^{13}$ President Elect, Intellectual Disability Section Royal Society Medicine, London, UK. ${ }^{14}$ University of the West of England, Bristol, UK. ${ }^{15}$ National Drug Research Institute Curtin University, Perth, Australia. ${ }^{16}$ East and North Hertfordshire NHS Trust, Hatfield, Hertfordshire, UK. ${ }^{17}$ East and North Hertfordshire NHS Trust. Centre for Child and Adolescent Mental Health, University College Ibadan, Hatfield, Hertfordshire, UK. ${ }^{18}$ FASD Specialist Behaviour Clinic, Surrey and Borders Partnership NHS Foundation Trust, Oxted, Surrey, UK. ${ }^{19}$ Sweden University, Department of Psychology Campus Östersund, Östersund, Sweden.
\end{abstract}

Received: 1 April 2016 Accepted: 31 August 2016

Published online: 22 September 2016

\section{References}

1. Polanczyk G, et al. The worldwide prevalence of ADHD: a systematic review and metaregression analysis. Am J Psychiatry. 2007;164(6):942-8.

2. Thapar, A., Cooper M., Attention deficit hyperactivity disorder. Lancet, Sept. 17, 2015. http://dx.doi.org/10.1016/S0140-6736(15)00238-X.

3. Peadon E, Elliott EJ. Distinguishing between attention-deficit hyperactivity and fetal alcohol spectrum disorders in children: clinical guidelines. Neuropsychiatr Dis Treat. 2010;6:509-15.

4. Burd L, Christensen T. Treatment of fetal alcohol spectrum disorders: are we ready yet? J Clin Psychopharmacol. 2009;29(1):1-4.

5. Rasmussen C, Bisanz J. Executive functioning in children with fetal alcohol spectrum disorders: profiles and age-related differences. Child Neuropsychol. 2009;15(3):201-15.

6. Gray R, Mukherjee R. A psychiatrist's guide to foetal alcohol spectrum disorders in mothers who drank heavily during pregnancy. Adv Ment Health Learn Disabil. 2007;1(3):19-26.

7. Spencer T, Biederman J, Mick E. Attention-deficit/hyperactivity disorder: diagnosis, lifespan, comorbidities, and neurobiology. J Pediatr Psychol. 2007;32(6):631-42.

8. American Psychiatric Association. The diagnostic and statistical manual of mental disorders. 5th ed. Arlington, VA: American Psychiatric Association. 2013.

9. Faraone SV, Biederman J, Mick E. The age-dependent decline of attention deficit hyperactivity disorder: a meta-analysis of follow-up studies. Psychol Med. 2006;36(2):159-65.

10. Weiss G, Hechtman LT. Hyperactive children grown up: ADHD in children, adolescents, and adults. New York: Guilford Press; 1993.

11. Thomas R, et al. Prevalence of attention-deficit/hyperactivity disorder: a systematic review and meta-analysis. Pediatrics. 2015;135(4):994-1001.

12. Valera $E M$, et al. Meta-analysis of structural imaging findings in attentiondeficit/hyperactivity disorder. Biol Psychiatry. 2007;61(12):1361-9.

13. Centers for Disease Control and Prevention. ADHD: Other Concerns and Conditions. Available from: www.cdc.gov/ncbddd/adhd/conditions.html. Accessed 15 Dec 2015.

14. Young $\mathrm{S}$, et al. A meta-analysis of the prevalence of attention deficit hyperactivity disorder in incarcerated populations. Psychol Med. 2015;45(2):247-58.

15. Young $\mathrm{S}$, et al. Co-morbid psychiatric disorders among incarcerated ADHD populations: a meta-analysis. Psychol Med. 2015;45(12):2499-510.
16. World Health Organization. International classification of diseases. 10th ed. Geneva: World Health Organization. 1992.

17. American Psychiatric Association. Diagnostic and statistical manual of mental disorders, text revision. 4th ed. Washington, DC: American Psychiatric Association. 2000

18. National Institute for Health and Clinical Excellence. Attention deficit hyperactivity disorder: Diagnosis and management of ADHD in children, young people and adults: NICE Guidelines [CG72]. 2008. Available from: https://www.nice.org.uk/ guidance/CG72/chapter/introduction. Accessed 1 July 2015.

19. British Medical Association; Board of Science. Fetal alcohol spectrum disorders: a guide for healthcare professionals. 2007.

20. Chudley AE, et al. Fetal alcohol spectrum disorder: Canadian guidelines for diagnosis. CMAJ. 2005;172(5 Suppl):S1-S21.

21. Jones KL, Smith DW. Recognition of the fetal alcohol syndrome in early infancy. Lancet. 1973;302(7836):999-1001.

22. Institute of Medicine: Committee to Study Fetal Alcohol Syndrome, Fetal Alcohol Syndrome: Diagnosis, Epidemiology, Prevention, and Treatment, ed. K. Stratton, C. Howe, and F.C. Battaglia. 1996, Washington D.C.: National Academies Press.

23. Streissguth AP, O'Malley K. Neuropsychiatric implications and long-term consequences of fetal alcohol spectrum disorders. Semin Clin Neuropsychiatry. 2000;5(3):177-90.

24. Crocker N, et al. Comparison of adaptive behavior in children with heavy prenatal alcohol exposure or attention-deficit/hyperactivity disorder. Alcohol Clin Exp Res. 2009;33(11):2015-23.

25. Fryer SL, et al. Evaluation of psychopathological conditions in children with heavy prenatal alcohol exposure. Pediatrics. 2007;119(3):e733-41.

26. Streissguth AP, et al. Risk factors for adverse life outcomes in fetal alcohol syndrome and fetal alcohol effects. J Dev Behav Pediatr. 2004;25(4):228-38.

27. National Organization on Fetal Alcohol Syndrome. FASD. Available from: http://www.nofas.org/about-fasd/. Accessed 4 July 2015.

28. May PA, Gossage JP. Estimating the prevalence of fetal alcohol syndrome. A summary. Alcohol Res Health. 2001;25(3):159-67.

29. May PA, et al. The epidemiology of fetal alcohol syndrome and partial FAS in a South African community. Drug Alcohol Depend. 2007:88(2-3):259-71.

30. May PA, et al. Prevalence and epidemiologic characteristics of FASD from various research methods with an emphasis on recent in-school studies. Dev Disabil Res Rev. 2009;15(3):176-92.

31. United States Census Bureau. U.S. and World Population Clock. Available from: www.census.gov/popclock/. Accessed 4 July 2016.

32. Lange $\mathrm{S}$, et al. Prevalence of fetal alcohol spectrum disorders in child care settings: a meta-analysis. Pediatrics. 2013;132(4):e980-95.

33. Chasnoff IJ, Wells AM, King L. Misdiagnosis and missed diagnoses in foster and adopted children with prenatal alcohol exposure. Pediatrics. 2015;135(2):264-70.

34. Gregory G, Reddy V, Young C. Identifying children who are at risk of FASD in Peterborough: working in a community clinic without access to gold standard diagnosis. Adopt Foster. 2015;39(3):225-34.

35. Spadoni AD, et al. Neuroimaging and fetal alcohol spectrum disorders. Neurosci Biobehav Rev. 2007;31(2):239-45.

36. Archibald SL, et al. Brain dysmorphology in individuals with severe prenatal alcohol exposure. Dev Med Child Neurol. 2001;43(3):148-54.

37. Popova S, et al. Comorbidity of fetal alcohol spectrum disorder: a systematic review and meta-analysis. Lancet. 2016;387(10022):978-87.

38. Brown NN, Gudjonsson G, Connor P. Suggestibility and fetal alcohol spectrum disorders: i'll tell you anything you want to hear. J Psychiatry Law. 2011;39(1):39-71.

39. Substance Abuse and Mental Health Services Administration. Results from the 2007 national survey on drug use and health: national findings. Rockville: Health and Human Services; 2008.

40. Floyd RL, et al. Recognition and prevention of fetal alcohol syndrome. Obstet Gynecol. 2005;106(5 Pt 1):1059-64.

41. Bertrand J, Floyd LL, Weber MK. Guidelines for identifying and referring persons with fetal alcohol syndrome. MMWR Recomm Rep. 2005;54(RR-11):1-14.

42. Kodituwakku PW. Defining the behavioral phenotype in children with fetal alcohol spectrum disorders: a review. Neurosci Biobehav Rev. 2007:31(2):192-201.

43. Olson $\mathrm{H}$. Advancing recognition of fetal alcohol spectrum disorders: the proposed DSM-5 diagnosis of "neurobehavioral disorder associated with prenatal alcohol exposure (ND-PAE)". Curr Dev Disord Rep. 2015;2(3):187-98. 
44. O'Malley KD. The knotted cord. Transgenerational alcohol related neurodevelopmental disorder (ARND). New York: Nova; 2014.

45. Glass $L$, et al. Neuropsychological deficits associated with heavy prenatal alcohol exposure are not exacerbated by comorbid adhd. Neuropsychology. 2013;27(6):713-24

46. Coles $C D$, et al. A comparison of children affected by prenatal alcohol exposure and attention deficit, hyperactivity disorder. Alcohol Clin Exp Res. 1997:21(1):150-61.

47. Crocker N, et al. Comparison of verbal learning and memory in children with heavy prenatal alcohol exposure or attention-deficit/hyperactivity disorder. Alcohol Clin Exp Res. 2011;35(6):1114-21.

48. Bhatara V. Association of attention deficit hyperactivity disorder and gestational alcohol exposure: an exploratory study. J Atten Disord. 2006;9(3):515-22.

49. Burd L, Carlson C, Kerbeshian J. Fetal alcohol spectrum disorders and mental illness. Int J Disabil Hum Dev. 2007:6(4):383-96.

50. O'Malley KD, Nanson J. Clinical implications of a link between fetal alcohol spectrum disorder and attention-deficit hyperactivity disorder. Can J Psychiatry. 2002;47(4):349-54.

51. Babor TF, Higgins-Biddle JC, Saunders JB, Monteiro MG. The Alcohol use disorders identifcation test. World Health Organization: Department of Mental Health and Substance Dependence. Geneva: 2001.

52. Li Q, et al. Fetal alcohol spectrum disorders: a population based study of premature mortality rates in the mothers. Matern Child Health J. 2012;16(6):1332-7.

53. Gudjonsson GH, et al. A national epidemiological study investigating risk factors for police interrogation and false confession among juveniles and young persons. Soc Psychiatry Psychiatr Epidemiol, 2015;51;359-367.

\section{Submit your next manuscript to BioMed Central and we will help you at every step:}

- We accept pre-submission inquiries

- Our selector tool helps you to find the most relevant journal

- We provide round the clock customer support

- Convenient online submission

- Thorough peer review

- Inclusion in PubMed and all major indexing services

- Maximum visibility for your research

Submit your manuscript at www.biomedcentral.com/submit 